\title{
The Utilization of Health Examination by Menopausal and Older Women - 6 Provinces, China, 2018
}

\author{
Bo Song'; Jiangli $\mathrm{Di}^{1, \text {,*; }}$ Gengli Zhao'; Yu Ma'; Linhong Wang,
}

\section{Summary}

What is already known about this topic?

Improving their utilization of health examination is important for improving the health of menopausal and older women.

What is added by this report?

Only $32.3 \%$ and $29.7 \%$ of women had been screened for cervical cancer and breast cancer, respectively. The overall utilization rate of health examination for menopausal and older women is low. The health examination services for menopausal and older women were utilized less in the western regions and in rural areas than in the eastern and central regions and in urban areas.

What are the implications for public health practice?

The imbalance of development is an important factor affecting the utilization of health examination for menopausal and older women. It is necessary to take effective measures to improve the level of service utilization in the western region and rural areas, in order to narrow the gap in health between different regions.

With the extension of human life expectancy and the intensification of the aging of the population, the number of menopausal and older women in China has increased sharply. According to the sixth census in 2010, the number of women aged 40-65 years old in China has reached 220 million (1). Menopause is a special period in a woman's life. With the gradual decline of ovarian function and the decline of hormone levels, menopausal women are prone to cardiovascular disease, diabetes, osteoporosis and other chronic diseases, which will affect their health and quality of life (2). Health examination is not only an important part of health care for menopausal and older women, but also an important means of detecting a variety of age related diseases in the early stages (3). In order to provide a scientific basis for targeted health care for older women and to promote the health of older women, a cross-sectional survey involving 5,049 women aged 50-70 years was conducted across eastern, central and western China. The main result of this study showed that the utilization rate of health examination for older women is low. The imbalance of development is an important factor affecting the utilization of health examination for menopausal and older women. The study was a cross-sectional survey in Jiangsu and Shandong, Hunan and Anhui, and Shaanxi and Sichuan provinces, which were selected to represent the three socio-economic regions of China: Eastern, Central and Western China. In each province one urban and one rural area was selected as investigation sites. Face-to-face interview questionnaires were completed by 5,049 women aged $50-70$ years in the 6 provinces.

The average age of the respondents was $58.94 \pm 6.195$ years. The main occupation of the cohort was farming, accounting for $55.2 \%$, followed by retirees, accounting for $17.1 \%$. Most of the participants $(55.0 \%)$ had only primary school education or were illiterate, followed by those who had junior and senior high school education, accounting for $42.0 \%$. The monthly income of the family was low - less than RMB 3,000 Yuan for 52.9\%, followed by RMB 3,000-4,999 Yuan for $27.1 \%$. The majority $(89.9 \%)$ of the women were postmenopausal (Table 1).

Among the 5,049 women, $46.4 \%$ (95\% CI: $45.0 \%-47.8 \%)$ of them reported never having undergone a regular physical examination (including examination of ultrasound, electrocardiogram, blood pressure, blood biochemistry, X-ray, bone mineral density, etc.) except for cervical cancer or breast cancer screening. The proportion in the western region $(52.3 \%, 95 \%$ CI: $49.8 \%-54.8 \%)$ was higher than that in the eastern region $(39.4 \%, 95 \%$ CI: $37.0 \%-41.8 \%)$ and the central region $(47.8 \%, 95 \% \mathrm{CI}$ : $45.4 \%-50.3 \%)$. There was significantly statistical difference among different regions $(p<0.001)$. The proportion of women who had had a regular physical examination within one year in the eastern region (40.9\%, 95\% CI: $38.5 \%-43.3 \%)$ was significantly 
higher than that in the central and western regions (28.2\%, 95\% CI: $26.0 \%-30.4 \%$ and $28.2 \%, 95 \%$ CI: $26.0 \%-30.5 \%$, respectively $) \quad(p<0.001)$. The proportion in rural areas $(36.8 \%$, 95\% CI: $34.9 \%-38.7 \%)$ was significantly higher than that in urban areas (28.1\%, 95\% CI: $26.3 \%-30.0 \%)$ $(p<0.001)$ (Table 2).

Of the 5,049 women, $64.2 \% \quad(95 \% \quad \mathrm{CI}$ : $62.9 \%-65.6 \%$ ) of them reported never having undergone breast or cervical cancer screening. The proportion of women who had never undergone breast or cervical cancer screening was highest in the western region $(73.9 \%, 95 \%$ CI: $71.8 \%-76.0 \%)$, followed by the central region $(60.5 \%, 95 \% \mathrm{CI}: 58.1 \%-62.9 \%)$ and the eastern region $(58.3 \%, 95 \% \quad \mathrm{CI}$ : $56.0 \%-60.7 \%) \quad(p<0.001)$. Only $27.8 \% \quad$ (95\% CI: $26.6 \%-29.0 \%$ ) of women reported having undergone both breast and cervical cancer screening. The proportion in the western region $(14.7 \%, 95 \% \mathrm{CI}$ : $13.0 \%-16.3 \%$ ) was significantly lower than that in the central (35.5\%, 95\% CI: 33.2\%-37.8\%) and eastern regions $(33.3 \%$, 95\% CI: $31.0 \%-35.5 \%)(p<0.001)$. The proportion in rural areas $(26.5 \%, 95 \% \mathrm{CI}$ : $24.8 \%-28.2 \%)$ was lower than that in urban areas (29.1\%, 95\% CI: $27.3 \%-30.9 \%)(p=0.015)$ (Table 2).

The proportion of women who had never undergone breast cancer screening $(70.3 \%, 95 \% \mathrm{CI}$ : $69.0 \%-71.6 \%$ ) was higher than the proportion of women who had never undergone cervical cancer screening $(67.7 \%, 95 \%$ CI: 66.4\%-68.9\%). The proportions of women in the western region who had never undergone cervical cancer screening $(77.6 \%$, 95\% CI: $75.6 \%-79.6 \%$ ) or breast cancer screening (83.8\%, 95\% CI: $82.0 \%-85.6 \%)$ were significantly higher than for those in the central $(63.2 \%, 95 \% \mathrm{CI}$ : $60.9 \%-65.5 \%$ and $62.5 \%$, 95\% CI: $60.2 \%-64.9 \%$, respectively) and the eastern regions $(62.3 \%, 95 \% \mathrm{CI}$ : 60.0\%-64.6\% and 64.6\%, 95\% CI: $62.4 \%-66.9 \%$ respectively) $(p<0.001)$. The proportion of women who had never been screened for cervical cancer in urban areas (69.6\%, 95\% CI: 67.8\%-71.4\%) was higher than that in rural areas $(65.8 \%, 95 \% \mathrm{CI}$ :

TABLE 1. Comparison of demographic characteristics of the respondents by region and area type -6 provinces, China, 2018.

\begin{tabular}{|c|c|c|c|c|c|c|}
\hline \multirow[b]{2}{*}{ Demographic characteristic } & \multirow{2}{*}{$\begin{array}{l}\text { Total } \\
\text { n (\%) }\end{array}$} & \multicolumn{3}{|c|}{ Region } & \multicolumn{2}{|c|}{ Area type } \\
\hline & & $\begin{array}{c}\text { Eastern } \\
\mathrm{n}(\%)\end{array}$ & $\begin{array}{c}\text { Central } \\
\mathrm{n}(\%)\end{array}$ & $\begin{array}{c}\text { Western } \\
\mathrm{n}(\%)\end{array}$ & $\begin{array}{l}\text { Urban } \\
\mathrm{n}(\%)\end{array}$ & $\begin{array}{l}\text { Rural } \\
\text { n (\%) }\end{array}$ \\
\hline \multicolumn{7}{|l|}{ Age (years) } \\
\hline $50-55$ & $1,949(38.6)$ & $675(39.6)$ & $669(40.1)$ & $605(36.0)$ & $915(36.3)$ & $1,034(40.9)$ \\
\hline $56-60$ & $968(19.2)$ & $333(19.6)$ & $328(19.7)$ & $307(18.3)$ & $502(19.9)$ & $466(18.4)$ \\
\hline $61-65$ & $1,140(22.6)$ & $367(21.6)$ & $367(22.0)$ & $406(24.2)$ & $612(24.3)$ & $528(20.9)$ \\
\hline$\geq 66$ & 992(19.6) & $328(19.3)$ & $303(18.2)$ & $361(21.5)$ & 494(19.6) & 498(19.7) \\
\hline \multicolumn{7}{|l|}{ Education degree } \\
\hline Primary school education or illiterate & $2,766(55.0)$ & $1,094(64.3)$ & $795(48.0)$ & $877(52.5)$ & $937(37.3)$ & $1,829(72.7)$ \\
\hline Junior and senior high school & $2,109(42.0)$ & $564(33.2)$ & $789(47.7)$ & $756(45.3)$ & $1,425(56.8)$ & $684(27.2)$ \\
\hline College or above & $151(3.0)$ & $43(2.5)$ & $71(4.3)$ & $37(2.2)$ & $147(5.9)$ & $4(0.2)$ \\
\hline \multicolumn{7}{|l|}{ Occupation } \\
\hline Farmers & $2,764(55.2)$ & $898(53.1)$ & $772(46.7)$ & $1,094(65.9)$ & $598(24.1)$ & $2,166(86.0)$ \\
\hline Retirees & $856(17.1)$ & $383(22.6)$ & $238(14.4)$ & $235(14.1)$ & $627(25.2)$ & $229(9.1)$ \\
\hline Workers & $470(9.4)$ & $211(12.5)$ & 152(9.2) & $107(6.4)$ & $424(17.1)$ & $46(1.8)$ \\
\hline Business and service staff & $244(4.9)$ & $68(4.0)$ & $122(7.4)$ & $54(3.3)$ & 197(7.9) & $47(1.9)$ \\
\hline Professional and technical personnel & $156(3.1)$ & $46(2.7)$ & $79(4.8)$ & $31(1.9)$ & $138(5.6)$ & $18(0.7)$ \\
\hline Others & $515(10.3)$ & $85(5.0)$ & $290(17.5)$ & $140(8.4)$ & $502(20.2)$ & $13(0.5)$ \\
\hline \multicolumn{7}{|l|}{ Monthly income of the family (RMB) } \\
\hline$<3,000$ & $2,644(52.9)$ & $731(43.2)$ & $744(44.8)$ & $1,169(71.1)$ & $1,062(42.7)$ & $1,582(63.0)$ \\
\hline $3,000-4,999$ & $1,355(27.1)$ & $479(28.3)$ & $537(32.3)$ & $339(20.6)$ & $768(30.9)$ & $587(23.4)$ \\
\hline $5,000-7,999$ & $586(11.7)$ & $244(14.4)$ & $250(15.1)$ & $92(5.6)$ & $379(15.2)$ & $207(8.2)$ \\
\hline$\geq 8,000$ & $412(8.2)$ & $237(14.0)$ & $130(7.8)$ & $45(2.7)$ & $277(11.1)$ & $135(5.4)$ \\
\hline
\end{tabular}


$63.9 \%-67.6 \%)(p=0.037)$. However, the proportion of women who had never had breast cancer screening in rural areas $(72.9 \%, 95 \%$ CI: $71.1 \%-74.6 \%)$ was significantly higher than in urban areas $(67.7 \%, 95 \%$ CI: $65.8 \%-69.5 \%)(p=0.001)$. (Table 2).

Of the reasons for not taking physical examination, absence of anybody to organize them accounted for the majority $(52.9 \%$, $95 \%$ CI: $50.8 \%-54.9 \%)$. The proportion of women in the western region reporting nobody to organize $(57.9 \%, 95 \%$ CI: $54.6 \%-61.2 \%)$ was higher than the proportions in the eastern $(47.6 \%$, 95\% CI: $43.7 \%-51.5 \%)$ and the central regions (51.7\%, 95\% CI: 48.2\%-55.3\%) $(p<0.001)$. The other reasons included thinking it was not necessary (25.1\%, 95\% CI: $23.4 \%-26.9 \%)$ and unwilling (18.5\%, 95\% CI: $16.9 \%-20.1 \%)$. The proportion of women who were unwilling in the eastern region (26.6\%, 95\% CI: $23.2 \%-30.0 \%)$ and rural areas (22.5\%, 95\% CI: $20.1 \%-25.0 \%)$ were higher than in the central $(17.2 \%, 95 \%$ CI: $14.5 \%-19.8 \%)$ and the western regions $(13.7 \%, 95 \% \mathrm{CI}: 11.4 \%-16.0 \%)$, and in urban areas (14.6\%, 95\% CI: $12.6 \%-16.7 \%)$ $(p<0.001)$ (Table 3).

Most of the women (53.7\%, 95\% CI: $51.9 \%-55.6 \%$ ) were organized by village committees or community workers to participate in physical examination. Only $27.5 \%$ (95\% CI: $25.8 \%-29.1 \%$ ) of them went to hospitals for physical examination on their own initiative. The proportion of women organized by village committees or community workers in the eastern region $(64.3 \%$, 95\% CI: $61.4 \%-67.2 \%)$ was significantly higher than that in the central (50.6\%, 95\% CI: $47.3 \%-53.9 \%)$ and the western regions $(43.9 \%$, 95\% CI: $40.5 \%-47.2 \%)(p<0.001)$. The proportion of women going to hospitals on their own initiative in urban areas $(35.9 \%, 95 \%$ CI: $33.3 \%-38.4 \%)$ was significantly higher than in the rural areas (19.0\%, 95\% CI: $16.9 \%-21.0 \%)$ $(p<0.001)$. (Table 3).

\section{DISCUSSION}

The overall utilization rate of health examination by older women was low. Breast and cervical cancer screening and regular physical examination are one of the important elements of health care for menopausal and older women. However, this study found that the utilization of health examination services by menopausal and older women was low. Only $32.5 \%$ of women had received a regular physical examination within one year, which was lower than the overall result of the Fifth National Health Service Survey (43.3\%) (4). Only $27.8 \%$ of women reported having been screened for both breast and cervical cancer in the past, and $32.3 \%$ and $29.7 \%$ of women had been screened for cervical cancer or breast cancer, respectively. The breast and cervical cancer screening rates were slightly higher than the results of the national sample survey in 2013 (22.5\% and 26.7\%, respectively) (5). However, the screening rate for breast $(16.4 \%)$ and cervical cancer $(15.2 \%)$ within one year were lower than those of the Fifth National Health Service Survey (26.5\% and 24.3\%, respectively) (4).

The village committee and community workers should play a greater role in organizing health examination to increase the participation rate. This study showed that a few (only 27.5\%) menopausal and older women took the initiative to obtain health examination services. Many studies have shown that the lack of awareness was a major obstacle for women to participate in the examination. Women with older age, low education level, underemployment, low family income and lack of health insurance are less likely to take the initiative to seek a health examination $(6-7)$. In this study, the most common reasons for not having a regular physical examination were the lack of organization, and feeling it was unnecessary or being unwilling. It reminds us that in order to enhance the service utilization of menopausal and older women and improve the participation rate, it is critical to encourage the village committee and community workers to play a role in organizing health examination, to carry out targeted health education for different groups of women.

The imbalance of regional economic development is an important factor affecting the utilization of services for menopausal and older women. Many studies have shown that the imbalance of regional economic development is an important factor restricting the utilization of services by menopausal and older women $(5,8)$. The results of this study showed that menopausal and older women in the western region and in rural areas were less likely to utilize the health examination services than those in the eastern and central regions and in urban areas. This pattern may be related to the health awareness, education level, economic conditions of women and level of development of the health system (7). Therefore, we need to take effective measures to effectively improve the level of service utilization in the western region and rural areas, in order to narrow the gap in health between different age groups of women. 


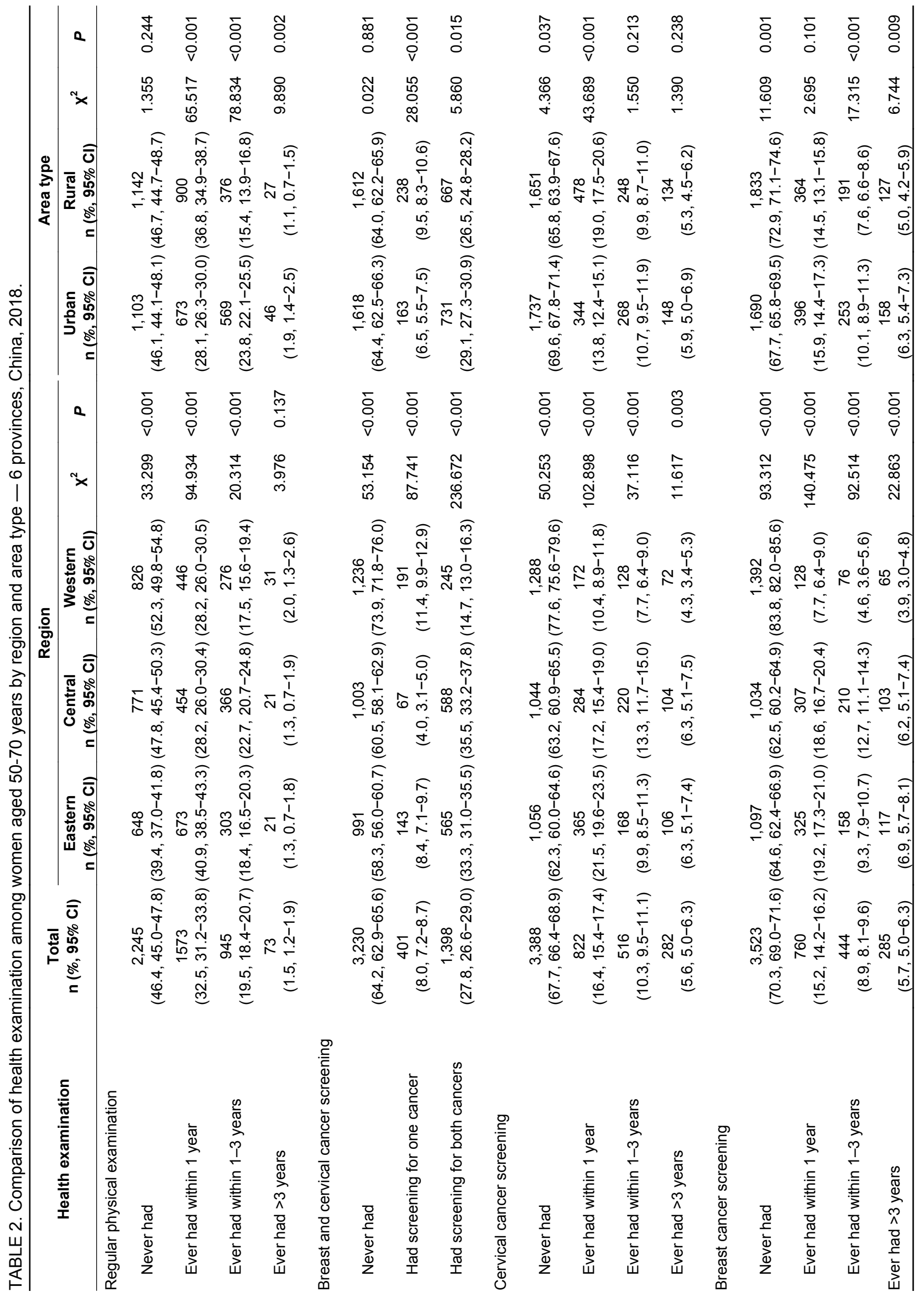




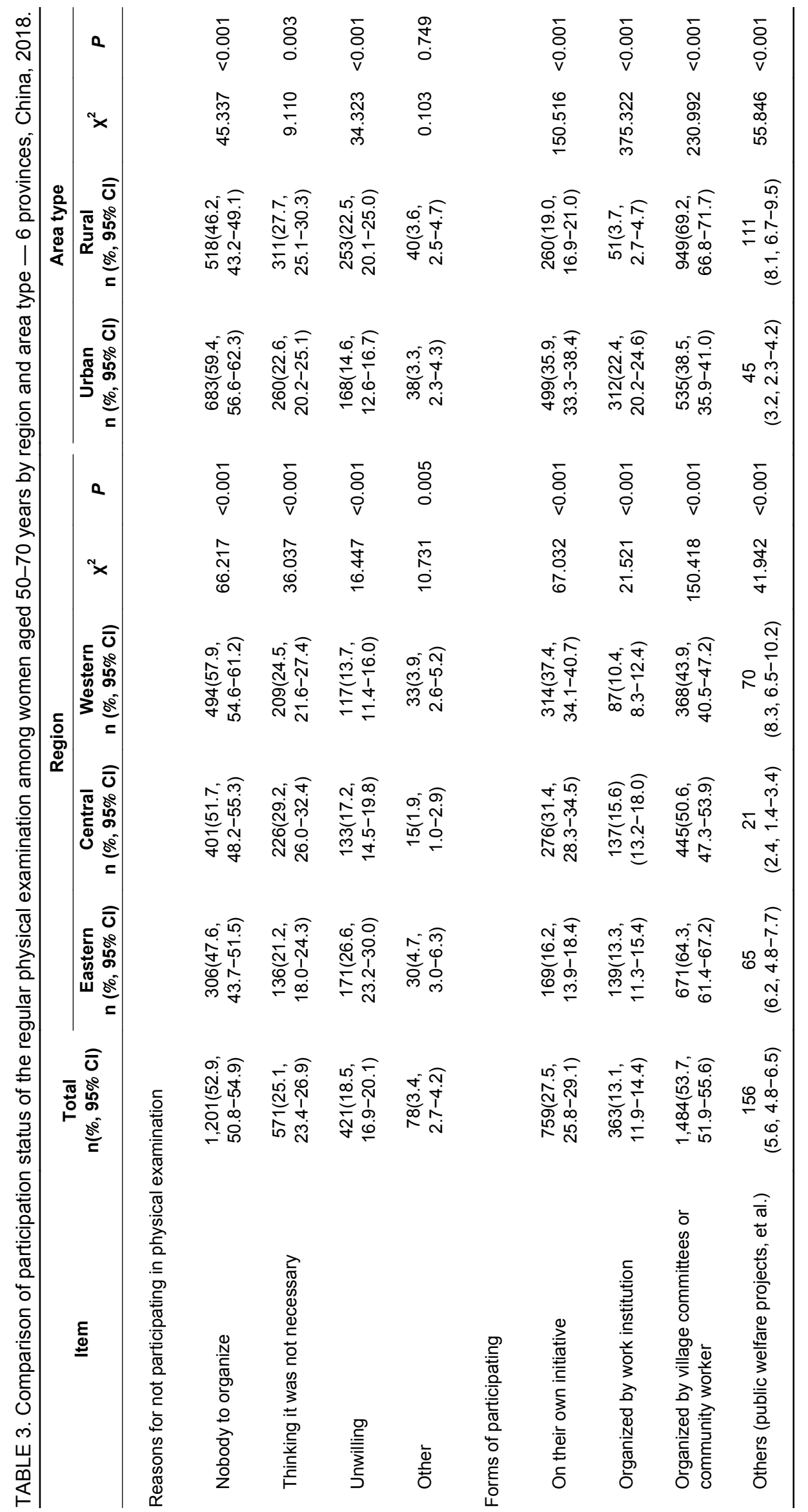


There were some limitations in this study. Firstly, self-reported information might be subjected to biases. Secondly, the study used convenience sampling, and data were collected in 12 counties/districts in 6 provinces, so the results might not be representative of the regional and national levels.

Acknowledgments: Appreciation is expressed to all the women who participated in the study. We also express our thanks to the efforts of all staff in the data collection in Jiangsu and Shandong, Hunan and Anhui, and Shaanxi and Sichuan provinces.

doi: $10.46234 / \mathrm{ccdcw} 2020.185$

* Corresponding authors: Jiangli Di, dijiangli@chinawch.org.cn; Linhong Wang, linhong@chinawch.org.cn.

\begin{abstract}
${ }^{1}$ National Center for Women and Children's Health, Chinese Center for Disease Control and Prevention, Beijing, China; ${ }^{2}$ Women and Children's Health Center of the First Hospital of Peking University, Beijing, China; ${ }^{3}$ National Center for Chronic and Non-communicable Disease Control and Prevention, Chinese Center for Disease Control and Prevention, Beijing, China.
\end{abstract}

Submitted: March 06, 2020; Accepted: July 16, 2020

\section{REFERENCES}

1. National Bureau of Statistics of the People's Republic of China. 2010 national census main data bulletin (No. 1). http://www.stats.gov. $\mathrm{cn} / \mathrm{tjsj} / \mathrm{pcsj} / \mathrm{rkpc} / 6 \mathrm{rp} / \mathrm{html} / \mathrm{fu} 03 . \mathrm{htm}$. [2020-02-12]. (In Chinese).

2. Ebtekar F, Dalvand S, Gheshlagh RG. The prevalence of metabolic syndrome in postmenopausal women: A systematic review and metaanalysis in Iran. Diabetes Metab Syndr 2018;12(6):955-60. http://dx.doi.org/10.1016/j.dsx.2018.06.002.

3. Women's Health Branch of Chinese Medical Association, Climacteric Health Care Group. Menopausal women's health care guide (2015). J PractGynecolEndocrinol2016;3(2):21 - 32.http://dx.doi.org/10.16484/j. cnki.issn2095-8803.2016.02.012. (In Chinese).

4. Center for Health Statistics and Information. An analysis report of national health services survey in China, 2013. http://www.nhc.gov. cn/mohwsbwstjxxzx/s8211/201610/9f109ff40e9346fca76dd82cecf419ce .shtml.[2020-02-12]. (In Chinese).

5. Bao HL, Wang LH, Wang LM, Fang LW, Zhang M, Zhao ZP, et al. Study on the coverage of cervical and breast cancer screening among women aged 35-69 years and related impact of socioeconomic factors in China, 2013. Chin J Epidemiol 2018;39(2):208 - 12. http://dx.doi.org/ 10.3760/cma.j.issn.0254-6450.2018.02.014. (In Chinese).

6. Ogunwale AN, Sangi-Haghpeykar H, Montealegre J, Cui YW, JibajaWeiss M, Anderson ML. Non-utilization of the Pap test among women with frequent health system contact. J Immigr Minor Health 2016;18(6):1404 - 12. http://dx.doi.org/10.1007/s10903-015-0287-9.

7. Romli R, Shahabudin S, Saddki N, Mokhtar N. Cervical cancer and pap smear screening: knowledge, attitude and practice among working women in northern state of Malaysia. Med J Malaysia 2019;74(1):8-14. https://pubmed.ncbi.nlm.nih.gov/30846655/.

8. Gong WY, Feng GY, Yuan F, Ding CC, Song C, Liu AL. Status of medical checkup among Chinese populations aged 15 years and above, 2010-2012. Chin J Public Health 2018;34(5):660 - 4. http://dx.doi.org/ 10.11847/zgggws1115351. (In Chinese). 